\title{
ASPECTOS TEMPORAIS AUDITIVOS: PRODUÇÃO DE CONHECIMENTO EM QUATRO PERIÓDICOS NACIONAIS
}

\author{
Temporal aspects of auditory: \\ knowledge production in four national journals
}

Sulamita da Silva Marcelino Terto ${ }^{(1)}$, Stela Maris Aguiar Lemos ${ }^{(2)}$

\begin{abstract}
RESUMO
Tema: o presente artigo foi produzido com base na revisão de artigos publicados desde o ano $2005 \mathrm{em}$ quatro periódicos nacionais importantes na área da Fonoaudiologia (Pró-Fono Revista de Atualização Científica, Revista Brasileira de Otorrinolaringologia, Revista CEFAC e Revista Sociedade Brasileira de Fonoaudiologia) a respeito dos aspectos temporais auditivos. Este estudo aponta para três temas abordados: Aspectos temporais, Ordenação temporal e Resolução temporal. Objetivo: delinear o perfil da produção de conhecimento na área de aspectos temporais auditivos tendo como referência quatro periódicos nacionais. Conclusão: o levantamento realizado nesta pesquisa permitiu verificar que existe um número reduzido de estudos nacionais relacionados ao tema sobre aspectos temporais auditivos. Considerando-se somente os quatro periódicos nacionais, menos de $9 \%$ das produções científicas abordaram essa temática.
\end{abstract}

DESCRITORES: Audição; Percepção Auditiva; Testes Auditivos

\section{INTRODUÇÃO}

A comunicação é uma habilidade fundamental ao ser humano. Por meio dela o homem integrase e interage com o seu semelhante e adquire linguagem mediante a recepção e transmissão de experiências e conhecimentos. Nessas circunstâncias, a audição possui função primordial ${ }^{1}$. Por isso, alterações que afetam a função auditiva podem influenciar diretamente na construção do complexo sistema de aprendizagem do homem ${ }^{2,3}$.

Sabe-se que a audição é um pré-requisito para o desenvolvimento da linguagem, uma vez que desempenha papel preponderante e decisivo na aquisição da fala e da escrita ${ }^{4}$. Contudo, para que um indivíduo receba, analise e interprete os sons, não basta possuir limiares auditivos normais, é

(') Acadêmica do curso de Fonoaudiologia da Universidade Federal de Minas Gerais - UFMG; Belo Horizonte, MG, Brasil.

(2) Fonoaudióloga; Professora Adjunto do Curso de Fonoaudiologia da Universidade Federal de Minas Gerais, UFMG, Belo Horizonte, MG, Brasil; Doutora em Distúrbios da Comunicação Humana (Fonoaudiologia) pela Universidade Federal de São Paulo, UNIFESP, Brasil.

Conflito de interesses: inexistente preciso que ele desenvolva um conjunto de habilidades específicas denominado Processamento auditivo (PA) ${ }^{5-7}$.

O Processamento auditivo é o termo usado para descrever a maneira como o cérebro reconhece e entende a informação auditiva que ouvimos ${ }^{8,9}$. Trata-se de um conjunto de habilidades auditivas realizadas pelo sistema nervoso central que são necessárias para interpretação dos sons ${ }^{6,10}$. É a compreensão do estímulo sonoro ${ }^{11}$.

Evidências sugerem que um elemento subjacente a inúmeras capacidades do processamento auditivo, são as habilidades do processamento temporal, uma vez que muitas das características que englobam a informação auditiva são de alguma forma, influenciadas pelo tempo ${ }^{6,12-14}$.

Logo, pode-se definir processamento auditivo temporal como a percepção do som ou da alteração do mesmo dentro de um concurso limitado ou definido de tempo ${ }^{13}$, ou seja, refere-se à habilidade de perceber ou diferenciar estímulos que são apresentados numa rápida sucessão ${ }^{6,12,15}$.

O processamento temporal de sinais auditivos pode ser dividido em quatro categorias que são críticas para as habilidades do processamento auditivo $^{13}$. Trata-se da ordenação ou sequencialização temporal, resolução, discriminação ou acuidade 
temporal, integração ou somação temporal e finalmente, o mascaramento temporal ${ }^{12}$. É importante ressaltar, que de acordo com $^{16}$ "estão disponíveis para a clínica somente medidas de avaliação para a ordenação e a resolução temporal, não havendo ainda medidas clinicamente viáveis para avaliar as outras duas categorias supracitadas".

A habilidade auditiva de ordenação temporal refere-se ao processamento de múltiplos estímulos auditivos em sua ordem de ocorrência no tempo ${ }^{1,12,15}$. Essa tem sido extensivamente investigada devido à sua importância na percepção da fala. Geralmente é avaliada clinicamente por meio dos testes de padrão de frequência ${ }^{5,11,17-20}$ e padrão de duração ${ }^{11,16,17,19,20}$.

A resolução, discriminação ou acuidade temporal refere-se habilidade do sistema auditivo em detectar dentro de um estímulo sonoro, mudanças rápidas e bruscas, ou seja, trata-se do intervalo de tempo mínimo necessário para a discriminação de eventos acústicos diferentes 3,5,6,12-15,21-23. O limiar de discriminação temporal é conhecido como acuidade auditiva temporal ou tempo de integração mínimo. Embora existam vários métodos para avaliar a resolução temporal, atualmente tem sido mais comumente realizados os testes AFT-R ${ }^{2,8,21}$, RGDT ${ }^{1,3,5,6,9,913,21-23}$ e GIN ${ }^{3,12-14,16,21}$.

As habilidades de ordenação e resolução temporais, de acordo com a literatura ${ }^{15,17}$ exercem funções essenciais na percepção da fala contínua e de suas partes isoladas, no aprendizado e na compreensão da linguagem, logo se constituem em pré-requisito para habilidades linguísticas, bem como na aquisição da leitura e escrita ${ }^{1}$.

Quando não desenvolvidas satisfatoriamente, essas habilidades podem trazer como consequências, indivíduos com dificuldades no processamento fonológico, na discriminação auditiva, na linguagem receptiva, na leitura, na escrita, na habilidade de soletrar, no aprendizado da linguagem oral, na segmentação fonêmica de palavras e no desenvolvimento de representações alteradas de informação verbal na memória. Pode ocorrer também, déficit na discriminação de frequência, intensidade e duração sonoras, assim como na prosódia e entonação das mensagens. A identificação das pequenas variações acústicas da fala pode estar prejudicada, logo, a produção da forma correta dos sons ficará comprometida $1,6,7,21-25$.

O aumento do interesse do processamento temporal como objeto de estudo é algo recente, especificamente na correlação das habilidades de resolução e ordenação temporais com a aprendizagem de uma maneira geral. Pode-se observar que estudos nacionais e internacionais relacionados a esse assunto ainda encontram-se em fases iniciais, tanto que, protocolos que estimam tais habilidades, ainda não correspondem à realidade da prática clínica na bateria de procedimentos que avaliam o Processamento Auditivo ${ }^{1,6}$. Porém, tendo-se conhecimento da importância do Processamento Temporal, pelo mesmo estar intimamente relacionado ao desenvolvimento adequado da linguagem ${ }^{14}$, este trabalho corrobora $\mathrm{com}^{23}$ quando indicam a necessidade de se incluir procedimentos que avaliem os aspectos temporais durante a avaliação do Processamento Auditivo 14,21,25 uma vez que conhecer as habilidades de resolução e ordenação temporais pode auxiliar na construção de programas de intervenção ${ }^{7,22}$.

Diante desses dados e com propósito de aprofundar o estudo de tais habilidades, incluindo os testes que as avaliam, assim como as variáveis que podem interferir nos resultados dos mesmos, optou-se pela realização de levantamento quanto ao estado da arte na área. Deste modo o presente estudo tem como objetivo delinear o perfil da produção de conhecimento na área de aspectos temporais auditivos tendo como referência quatro periódicos nacionais.

\section{MÉTODO}

Trata-se de revisão integrativa de literatura realizada em duas etapas. Foram utilizados artigos das seguintes bases de dados da internet: Bireme, Lilacs, SciELO, publicados nos últimos cinco anos e acessados até a data limite de Julho de 2010.

$\mathrm{Na}$ primeira etapa da pesquisa, a busca por artigos foi feita por meio de três descritores: audição, percepção auditiva, testes auditivos. Nas bases de dados Lilacs e SciELO , a busca foi feita por meio da associação do descritor percepção auditiva com cada um dos outros três descritores, e foram selecionados os artigos publicados no intervalo de tempo estabelecido. Na base de dados Bireme, a busca foi feita para cada um dos três descritores, e os artigos foram encontrados por meio do refinamento dos resultados por: 1. Tipo de publicação: artigo; 2. Limites da publicação: humanos; 3. Idioma: português e inglês; 4. Ano de publicação: 2005, 2006, 2007, 2008, 2009 e 2010.

$\mathrm{Na}$ segunda etapa da pesquisa, foram excluídos todos os artigos cujos textos não estivessem disponíveis, na íntegra, na(s) base(s) de dados em que foram encontrados.

Respeitando-se o limite de 60 referências bibliográficas estabelecido pelo presente periódico, foram recuperados 32 artigos sobre ordenação e resolução temporais que atenderam aos objetivos desta revisão. Durante a análise e seleção, foram considerados as informações contidas nos 
textos, consistência e os dados apresentados pelos autores. Seguem informações sobre os periódicos selecionados, baseado na ordem cronológica de criação.

A Pró-Fono Revista de Atualização Científica foi fundada em 1989 e recebe apoio financeiro do Conselho Nacional de Desenvolvimento Científico e Tecnológico (CNPq) desde 2001. A mesma é indexada nas seguintes Bases de Dados: Index Medicus e MEDLINE, PubMed, desde 2004; Scopus, desde 2008; SciELO; desde 2005; CSA, desde 2000; LILACS, desde 1990; Portal de Periódicos Capes, desde 2005; CECAE, desde 2003.

A Revista Brasileira de Otorrinolaringologia, criada originalmente em 1993, com o nome de "Revista Oto-laryngológica de São Paulo" e atualmente com o título de Brasilian Journal of Otorhinolaryngology, na versão impressa, possui como objetivo "a divulgação em âmbito nacional e internacional da produção científica de interesse da otorrinolaringologia, bem como a discussão, em editoriais de temas de importância científica, acadêmica e profissional da especialidade". As áreas de interesse são a otorrinolaringologia e áreas de atuação a ela associadas. O periódico é indexado ao SciELo desde 2001, à LILACS e ao MEDLINE desde 2005.

A Revista CEFAC foi criada em 1999. Esse periódico pretende construir um instrumento de comunicação e divulgação científica na área da Fonoaudiologia nos territórios nacional e internacional. Para tanto, suas publicações visam "contemplar os critérios de avaliação adotados pela CAPES para composição do Qualis, assim como as indexações". Em 2006, a revista foi indexada na Base de dados LILACS e coleção SciELO Brasil. Atualmente são publicados 20 artigos científicos em cada número, organizados por áreas de abrangência da revista: Linguagem, Motricidade Orofacial, Audiologia, Voz, Saúde Coletiva, Educação e áreas afins.

Por último, a Revista Sociedade Brasileira de Fonoaudiologia apresentou sua primeira publicação em junho de 2007. A indexação desta visa atender às necessidades dos pesquisadores da área para divulgação de seus trabalhos e às exigências dos órgãos de avaliação da produção científica nacional. Em 2006, o periódico foi indexado na ScieLO.

A Revista possui como objetivo "divulgar a produção científica sobre temas relevantes de Fonoaudiologia, Distúrbios da Comunicação Humana e áreas afins".

\section{REVISÃO DE LITERATURA}

\section{Aspectos temporais}

O interesse pelo papel dos aspectos temporais auditivos foi originado pela constatação de sua atribuição fundamental na habilidade da comunicação humana. Os aspectos temporais auditivos estão relacionados intimamente na capacidade do indivíduo em reconhecer, discernir e perceber os aspectos segmentais e suprassegmentais da fala ${ }^{1,5}$. Além disso, sabe-se que a integridade desses aspectos é um pré-requisito para que o sistema auditivo defina a duração dos sons, o intervalo que os separa e a sequência em que ocorrem, habilidades essas, imprescindíveis para o processamento da música e da fala ${ }^{13,15,23}$.

Os aspectos temporais podem ser divididos em categorias que auxiliam no entendimento de alguns dos mecanismos e processos do sistema nervoso central. A ordenação e a resolução temporal, duas dessas categorias ${ }^{1}$, contribuem de maneira relevante para o desenvolvimento da fala, logo, alterações nessas habilidades, tornam possível a presença de problemas na comunicação.

\section{Ordenação Temporal}

A ordenação ou sequencialização temporal refere-se à capacidade de reconhecer, identificar e ordenar estímulos acústicos, de acordo com sua ordem de apresentação, durante determinado período de tempo. As áreas envolvidas nesse processo estariam possivelmente localizadas nos lobos temporais do cérebro (principalmente giro de Herschl) ${ }^{16}$. Tal habilidade, em que muitos processos perceptivos e cognitivos participam, é considerada uma das funções mais importantes do sistema nervoso central, uma vez que a fala e a compreensão da linguagem, são dependentes da capacidade de se trabalhar com a sequência sonora ${ }^{19}$.

Sabe-se que os testes responsáveis pela avaliação da ordenação temporal foram desenvolvidos na década de 60, com o propósito de conhecer as disfunções do Sistema Nervoso Central ${ }^{19}$.

A literatura descreve diversos estudos que utilizaram os Testes Tonais de Padrão de Frequência - Pitch Pattern Test (TPF) 5,10,11,17-20,26-28 e os Testes Tonais de Padrão de Duração do Som - Duration Pattern Test (TPD) ${ }^{7,11,16,17,19,20,28-30}$ para avaliação da ordenação temporal.

A maioria destes estudos correlacionou os resultados dos testes supracitados 11,17,19,20,28. Foi permitido constatar que até os 12 anos de idade, os resultados de ambos os testes são concordantes ${ }^{19}$. A partir dessa idade, observa-se independência nos resultados dos testes. Há relato de indivíduos com desempenho superior no TPF ${ }^{11}$, e estudos que relatam desempenho superior no TPD ${ }^{11,19}$.

Em um estudo com descendentes de japoneses falantes e não-falantes da língua japonesa e indivíduos não-orientais, foram comparados os desempenhos no reconhecimento de padrões temporais. 
Os autores constataram que a educação musical não atua no desempenho do TPD, porém influencia na capacidade de discriminação de frequência, uma vez que os indivíduos não-orientais (possuidores de alguma habilidade musical) apresentaram uma média maior de acertos com significância estatística, quando comparado ao desempenho dos indivíduos não-orientais ${ }^{11}$. Este achado corrobora com o resultado de outro estudo ${ }^{5}$, no qual o TPF, em um estudo com cantores profissionais e amadores afinados e desafinados, sofreu influência da variável estudo de teoria musical, pois neste teste os cantores profissionais apresentaram desempenho estatisticamente superior em relação aos cantores amadores. Esses autores também observaram que os resultados das orelhas testadas (direita e esquerda) não diferiram com significância estatística durante a aplicação do TPF.

Porém, em outro estudo, cujo objetivo consistia em comparar o desempenho de crianças com e sem prejuízo na Prova de Consciência Fonológica nos testes TPF e TPD, os autores relataram ter observado vantagem da orelha esquerda em relação à direita no teste TPF. A conclusão do estudo relata que crianças com déficit em consciência fonológica apresentaram alteração na ordenação temporal ${ }^{17}$.

Sabe-se por meio da literatura especializada que a percepção dos aspectos temporais do som desempenha papel crucial na aquisição da leitura e da escrita ${ }^{17}$. Há correlação entre os processamentos lingüístico e auditivo temporal em crianças com distúrbio específico de linguagem (DEL). O desempenho de crianças com DEL no teste de TPF está diretamente relacionado ao grau de dificuldade das tarefas de complexidade sintática, ou seja, quanto maior for a dificuldade em processamento sintático, pior será o desempenho da criança no teste. Isso porque o processamento sintático é altamente dependente das relações de processamento temporal ${ }^{18}$. Os autores acrescentam ainda que, o baixo desempenho no TPF pode ser um indicativo de déficit em processamento lingüístico complexo, ressaltando a importância do teste no diagnóstico dessas crianças.

O desempenho de indivíduos adultos usuários de implante coclear (IC) nos testes que avaliam a ordenação temporal foi objeto de estudo ${ }^{20}$. Foi observado que usuários de implante coclear apresentaram desempenho semelhante nos testes TPF e TPD quando comparados aos indivíduos com audição normal. Os autores relatam ainda que, a utilização de testes auditivos comportamentais pós-cirurgia de implante coclear poderá auxiliar na análise do desempenho do indivíduo em relação à percepção de fala, contudo, enfatizam que, as outras variáveis relacionadas ao implante coclear não devem ser desconsideradas (etiologia da perda auditiva, o modelo do IC e outras).

Atualmente, os testes comportamentais têm se mostrado instrumento muito útil na avaliação do processamento temporal, uma vez que proporcionam maiores informações sobre déficits funcionais da percepção auditiva, descrevendo possíveis alterações. Por isso conhecê-los torna-se imprescindível na prática clínica ${ }^{19,22}$. Um estudo buscou verificar a concordância entre os resultados dos testes que avaliam a ordenação temporal e o Potencial Evocado Auditivo de Média Latência $(P E A M L)^{19}$. Nesse trabalho os autores, mesmo não encontrando concordâncias entre o PEAML e os testes TPF e TPD, concluíram que a avaliação da percepção auditiva pode tornar-se mais eficiente ao se associarem testes eletrofisiológicos aos comportamentais, isso porque os primeiros testes são capazes de identificar alterações do processamento auditivo, revelando integridade e capacidade do sistema nervoso central e os outros testes, são capazes de descrever as alterações ou déficits funcionais.

\section{Resolução temporal}

A capacidade de perceber mudanças acústicas transitórias é essencial para compreensão da fala humana, visto que vários padrões dos sons da fala distinguem-se por diferenças temporais de poucos milissegundos. A habilidade de resolução temporal é responsável por identificar tais diferenças temporais, pois refere-se ao tempo mínimo necessário ao sistema auditivo para determinar ou separar eventos acústicos ocorridos em um curto intervalo de tempo ${ }^{12}$.

A análise da resolução temporal data a partir da década de 70 , porém os testes utilizados para investigá-la tornaram-se comercialmente disponíveis apenas no final da década de $90^{6}$.

Dentre os procedimentos utilizados para avaliação da resolução temporal existe o teste de fusão auditiva revisado (Auditory Fusion TestRevised-AFT-R) ${ }^{6,8}$. Este teste mensura o limiar de fusão auditiva, medido em milissegundos (ms), por meio da identificação de estímulos sonoros relacionados à duração do intervalo de silêncio entre os pares apresentados no teste ${ }^{21}$. Os intervalos de silêncio são apresentados em ordem crescente e decrescente de duração, em tons puros nas frequências de 250 a $4000 \mathrm{~Hz}$, no qual o indivíduo deve indicar se detectou um ou dois tons ${ }^{2,23}$. O AFT-R foi utilizado em pesquisa com crianças com fissura palatina ${ }^{2}$ comprovando que os limiares de fusão auditivos são maiores se comparados às crianças sem fissura, sugerindo alteração no processamento auditivo temporal. A justificativa para tal achado é 
atribuída à recorrência de episódios de otite média nessas crianças. Outra pesquisa com crianças com fissura labiopalatina investigou o processamento temporal auditivo por meio de uma bateria de testes, entre eles, o AFT-R. Foi constatado que uma alta porcentagem de crianças do estudo apresentou piores desempenhos em diversos testes, no qual o teste de Fusão Auditiva Revisado estava incluído8.

Após algumas pesquisas utilizando o AFT-R, foi verificada a necessidade de tornar sua aplicação mais rápida, além de eliminar a possibilidade de predição das respostas ${ }^{23}$. Consequentemente foi desenvolvida uma revisão desse teste, que permitiu apresentar os intervalos de silêncio aleatoriamente, ou seja, randomizada. Trata-se do RGDT (Random Gap Detection Test) ${ }^{6,21}$, que consiste em apresentar tons puros pareados com pequenos intervalos de silêncio, com variação de zero a $40 \mathrm{~ms}$. Sendo considerado como limiar de detecção, o menor intervalo no qual foi identificado a ocorrência de dois estímulos ${ }^{21,23}$. Na literatura, foram encontradas diversas pesquisas que utilizaram o RGDT para avaliação do processamento temporal em crianças nascidas a termo e pré-termo ${ }^{22}$; escolares com desenvolvimento normal ${ }^{3}$; crianças $\operatorname{com}^{15} \mathrm{e}$ sem desvio fonológico ${ }^{1}$; mulheres jovens e idosas ${ }^{23}$; crianças portadoras de perda auditiva condutiva e crianças com distúrbio do processamento auditivo ${ }^{6}$; operadores de telemarketing ${ }^{9}$ e adultos jovens normais ${ }^{13}$. Os estudos em sua maioria, tiveram grupo controle que apresentou resultados melhores que o grupo caso no RGDT 1,6,22,23.

Além dos procedimentos supracitados, existe ainda o Teste de Detecção de Gaps no Ruído (Gaps in noise - GIN). Nesse, apresenta-se seis interrupções de diferentes durações (2 a 20 ms) ao longo da apresentação de um ruído. O limiar de detecção é obtido a partir do menor intervalo detectado em quatro das seis apresentações em cada segmento de ruído branco apresentado no teste ${ }^{13,21}$. Sabe-se que o teste GIN foi publicado em $2005^{3}$, devido a isso esse protocolo apenas foi utilizado em crianças normais, visando o estabelecimento de critérios de normalidade ${ }^{14} \mathrm{e}$ em adultos, para comparação de desempenho das orelhas direita e esquerda. Foi constatado que não há diferença nos limiares de detecção de gap, nem na porcentagem de acertos entre as orelhas direita e esquerda ${ }^{31}$.

$\mathrm{Na}$ literatura existem diversos estudos que compararam os dois protocolos de avaliação da resolução temporal (GIN e RGDT) ${ }^{3,13,21}$. Todos os estudos assentiram que a natureza de investigação dos testes GIN e RGDT é distinta, uma vez que existe diferença entre os limiares de detecção obtidos na mesma amostra. Tais disparidades são ocasionadas pela diferença da natureza do estímulo e da tarefa solicitada ${ }^{3,13,21}$. Uma outra pesquisa ${ }^{31}$ afirma que os tipos de marcadores, posicionamento dos gaps, intensidade, frequência, apresentação do estímulo e efeitos do rise (tempo de surgimento do sinal) e do fall (tempo de declínio do sinal) podem influenciar os limiares de detecção dos gaps ${ }^{21}$.

Existem ainda estudos sobre a influência dos paradigmas temporais em testes de processamento temporal auditivo ${ }^{25,32}$. Em uma pesquisa ${ }^{25}$ constatou-se que as variáveis duração do estímulo e ordem emitida pelo avaliador podem interferir no desempenho dos testes. Em pesquisa posterior ${ }^{32}$ correlacionaram-se leitura, consciência fonológica e processamento temporal auditivo em crianças com e sem dislexia. Foi constatado que as crianças com dislexia apresentaram desempenho inferior em todos os testes quando comparadas ao grupo controle, contudo a dificuldade nas tarefas do processamento temporal auditivo não foi correlacionada com o desempenho das tarefas de leitura e consciência fonológica.

Para melhor compreensão da pesquisa, optouse por organizar a apresentação dos periódicos por ordem cronológica de criação. Portanto segue a ordem descrita a seguir:

O período Pró-Fono Revista de Atualização Científica, entre janeiro de 2005 e junho de 2010, realizou 21 publicações em números que totalizaram em 262 produções. Dessas, 13 (4,96\%) citaram um dos aspectos temporais auditivos como tema.

A Figura 1 resume o tema, os métodos utilizados e as conclusões destes estudos.

A Revista Brasileira de Otorrinolaringologia, no período entre janeiro de 2005 e junho de 2010, publicou 34 números, o que corresponde a um total de 803 produções. Dessas, 08 (0,99\%) referenciaram um dos aspectos temporais auditivos como tema.

A Figura 2 sintetiza o tema, os métodos utilizados e as conclusões dos estudos encontrados.

A Revista CEFAC, no período entre janeiro de 2005 e abril de 2010, realizou 26 publicações em números que totalizaram em 475 produções. Sendo 6 delas $(1,26 \%)$ a respeito de um dos aspectos temporais auditivos.

A Figura 3 sintetiza o tema, os métodos utilizados e as conclusões dos estudos encontrados.

Por último, a Revista Sociedade Brasileira de Fonoaudiologia, entre janeiro de 2006 e junho de 2010, publicou 17 números, totalizando 299 produções. Entre elas artigos científicos originais, relatos de caso, artigos de revisão, resenhas e resumos dos quais 4 dessas publicações $(1,33 \%)$ apresentaram um dos aspectos temporais auditivos como tema. 


\begin{tabular}{|c|c|c|c|c|}
\hline & $\mathbf{N}$ & Tema & Métodos & Conclusões \\
\hline $\begin{array}{l}\text { Ramos, } \\
\text { Pereira, } 2005\end{array}$ & 32 & $\begin{array}{l}\text { Processamento auditivo } \\
\text { e sensibilidade auditiva } \\
\text { para altas frequências } \\
\text { em crianças. }\end{array}$ & $\begin{array}{l}\text { Realização da ASPA, TPD e } \\
\text { Audiometria de alta } \\
\text { frequência. }\end{array}$ & $\begin{array}{l}\text { Indícios inconclusivos sobre a } \\
\text { relação entre os limiares de } \\
\text { audibilidade aumentados e alteração } \\
\text { do processamento auditivo. }\end{array}$ \\
\hline $\begin{array}{l}\text { Ishii et al., } \\
2006\end{array}$ & 78 & $\begin{array}{l}\text { Desempenho de } \\
\text { cantores profissionais a } \\
\text { amadores afinados e } \\
\text { desafinados no teste } \\
\text { RGDT. }\end{array}$ & $\begin{array}{l}\text { Questionário e realização do } \\
\text { TPF e RGDT. }\end{array}$ & $\begin{array}{l}\text { O TPF mostrou-se sensível para } \\
\text { distinguir cantores profissionais de } \\
\text { amadores, ao contrário do RGDT. }\end{array}$ \\
\hline $\begin{array}{l}\text { Elias et al., } \\
2007\end{array}$ & 01 & $\begin{array}{l}\text { Manifestações } \\
\text { audiológicas de uma } \\
\text { criança com doença } \\
\text { cerebrovascular em dois } \\
\text { momentos distintos da } \\
\text { evolução clínica. }\end{array}$ & $\begin{array}{l}\text { Realização de Avaliação } \\
\text { Simplificada do } \\
\text { Processamento Auditivo e } \\
\text { testes: FR, FF, DNV, DCV, } \\
\text { DD, SSW e TPF. }\end{array}$ & $\begin{array}{l}\text { A evolução, embora favorável, } \\
\text { mostrou-se aquém do esperado } \\
\text { para idade quando comparado ao } \\
\text { seu par. }\end{array}$ \\
\hline $\begin{array}{l}\text { Fortes et al., } \\
2007\end{array}$ & 70 & $\begin{array}{l}\text { Crianças nascidas pré- } \\
\text { termo e crianças } \\
\text { nascidas a termo no } \\
\text { teste RGDT. }\end{array}$ & Utilização do Teste RGDT. & $\begin{array}{l}\text { As crianças nascidas pré-termo } \\
\text { apresentaram a habilidade de } \\
\text { resolução temporal inferior se } \\
\text { comparada às crianças nascidas a } \\
\text { termo. }\end{array}$ \\
\hline $\begin{array}{l}\text { Murphy, } \\
\text { Schochat, } \\
2007\end{array}$ & 27 & $\begin{array}{l}\text { Crianças em testes de } \\
\text { processamento temporal } \\
\text { auditivo de acordo com } \\
\text { diferentes paradigmas } \\
\text { temporais. }\end{array}$ & $\begin{array}{l}\text { Utilização adaptação do } \\
\text { teste americano “Repetition } \\
\text { Test”. }\end{array}$ & $\begin{array}{l}\text { Duas variáveis temporais (duração } \\
\text { do estímulo e ordem solicitada) } \\
\text { podem interferir no desempenho de } \\
\text { crianças. }\end{array}$ \\
\hline $\begin{array}{l}\text { Zaidan et al., } \\
2008\end{array}$ & 25 & $\begin{array}{l}\text { Adultos jovens normais } \\
\text { nos testes GIN e RGDT. }\end{array}$ & $\begin{array}{l}\text { Realização dos testes GIN e } \\
\text { RGDT. }\end{array}$ & $\begin{array}{l}\text { O sexo masculino obteve melhor } \\
\text { desempenho nos dois testes. } \\
\text { Porém, os dois sexos apresentaram } \\
\text { desempenho melhor no teste GIN. }\end{array}$ \\
\hline $\begin{array}{l}\text { Murphy } \\
\text { Schochat, } \\
2009\end{array}$ & 66 & $\begin{array}{l}\text { Crianças com dislexia } \\
\text { nas habilidades de } \\
\text { leitura, consciência } \\
\text { fonológica e } \\
\text { processamento auditivo } \\
\text { temporal. }\end{array}$ & $\begin{array}{l}\text { Utilização do teste de leitura } \\
\text { de palavras isoladas } \\
\text { (adaptação); teste de } \\
\text { consciência fonológica e } \\
\text { teste "Repetition Test". }\end{array}$ & $\begin{array}{l}\text { As crianças com dislexia } \\
\text { apresentaram desempenho pobre } \\
\text { no teste de resolução temporal } \\
\text { auditivo, contudo não foi possível } \\
\text { relacioná-lo ao desempenho dos } \\
\text { outros dois testes. }\end{array}$ \\
\hline $\begin{array}{l}\text { Fortunato- } \\
\text { Tavares et } \\
\text { al., } 2009\end{array}$ & 23 & $\begin{array}{l}\text { Processamento } \\
\text { Temporal (TPF) e } \\
\text { Processamento } \\
\text { Linguístico (Teste de } \\
\text { Compreensão Sintática). }\end{array}$ & $\begin{array}{l}\text { Utilização dos testes de } \\
\text { Compreensão Sintática e } \\
\text { TPF. }\end{array}$ & $\begin{array}{l}\text { O TFP está correlacionado } \\
\text { positivamente com habilidades de } \\
\text { complexidade sintática. }\end{array}$ \\
\hline $\begin{array}{l}\text { Perez, } \\
\text { Pereira, } 2010\end{array}$ & 92 & $\begin{array}{l}\text { Critérios de normalidade } \\
\text { para o teste GIN em } \\
\text { crianças de } 11 \text { e } 12 \\
\text { anos. }\end{array}$ & Utilização do teste GIN. & $\begin{array}{l}\text { O teste não sofreu influência } \\
\text { estatisticamente significante das } \\
\text { variáveis faixa etária, orelha e } \\
\text { gênero. Em } 78,27 \% \text { da população } \\
\text { do estudo, as respostas } \\
\text { encontradas são recomendadas } \\
\text { como referência de normalidade } \\
\text { para a faixa etária. }\end{array}$ \\
\hline $\begin{array}{l}\text { Abdo et al., } \\
2010\end{array}$ & 30 & $\begin{array}{l}\text { Crianças com dislexia e } \\
\text { TDAH em testes } \\
\text { comportamentais do } \\
\text { processamento auditivo. }\end{array}$ & $\begin{array}{l}\text { Utilização dos testes FR, DD } \\
\text { e TPF. }\end{array}$ & $\begin{array}{l}\text { O grupo com TDAH apresentou pior } \\
\text { desempenho, seguido do grupo com } \\
\text { dislexia em todos os testes } \\
\text { aplicados. }\end{array}$ \\
\hline $\begin{array}{l}\text { Frederigue- } \\
\text { Lopes et al., } \\
2010 .\end{array}$ & 43 & $\begin{array}{l}\text { Crianças com audição } \\
\text { normal no TPF e TPD. }\end{array}$ & $\begin{array}{l}\text { Utilização dos testes TPF e } \\
\text { TPD. }\end{array}$ & $\begin{array}{l}\text { Os valores obtidos para os dois } \\
\text { testes podem ser considerados } \\
\text { como referência para versão infantil } \\
\text { em campo livre. }\end{array}$ \\
\hline
\end{tabular}

Legenda: ASPA= Avaliação Simplificada do Processamento Auditivo; TPD= Teste Padrão de Duração; TPF= Teste Padrão de Frequência; RGDT= Random Gap Detection Test; FR= Fala com Ruído; FF= Fala Filtrada; DNV= Dicótico não-verbal; DCV= Dicótico Consoante-vogal; DD= Dicótico de Dígitos; SSW= Staggered Spondaic Word Test; GIN= Gaps in Noise e TDAH= Transtorno de Déficit de Atenção e Hiperatividade.

Figura 1 - Publicações sobre aspectos temporais auditivos no Periódico Pró-Fono Revista de Atualização Científica 


\begin{tabular}{|c|c|c|c|c|}
\hline & $\mathbf{N}$ & Tema & Métodos & Conclusões \\
\hline $\begin{array}{l}\text { Onoda et al., } \\
2006\end{array}$ & 60 & $\begin{array}{l}\text { Comportamento auditivo } \\
\text { temporal e escuta } \\
\text { dicótica em } \\
\text { descendentes de } \\
\text { japoneses, falantes ou } \\
\text { não-falantes da língua } \\
\text { japonesa e brasileiros. }\end{array}$ & $\begin{array}{l}\text { Utilização de questionário e } \\
\text { dos testes: TPF, TPD, SSW } \\
\text { e DD. }\end{array}$ & $\begin{array}{l}\text { Os descendentes de japoneses } \\
\text { falantes ou não da língua } \\
\text { japonesa apresentaram } \\
\text { desempenho melhor no TPF se } \\
\text { comparados aos brasileiros e } \\
\text { quando comparados ao } \\
\text { desempenho no TPD. }\end{array}$ \\
\hline $\begin{array}{l}\text { Cassab, } \\
\text { Zorzeto, } \\
2006\end{array}$ & 55 & $\begin{array}{l}\text { Teste AFT-R em crianças } \\
\text { com fissura labiopalatina. }\end{array}$ & Realização do Teste AFT-R. & $\begin{array}{l}\text { Crianças com fissura } \\
\text { labiopalatina apresentaram } \\
\text { limiares maiores no AFT-R } \\
\text { quando comparados aos das } \\
\text { crianças sem fissura. }\end{array}$ \\
\hline $\begin{array}{l}\text { Meneguello } \\
\text { et al., } 2006\end{array}$ & 08 & $\begin{array}{l}\text { Processamento auditivo } \\
\text { em indivíduos com } \\
\text { epilepsia de lobo } \\
\text { temporal. }\end{array}$ & $\begin{array}{l}\text { Utilização dos testes de LS, } \\
\text { TPD, DD e DNV. }\end{array}$ & $\begin{array}{l}\text { Indivíduos com epilepsia do lobo } \\
\text { temporal apresentaram } \\
\text { desempenho inferior nos testes } \\
\text { de processamento auditivo, } \\
\text { exceto na discriminação da } \\
\text { fonte sonora. }\end{array}$ \\
\hline $\begin{array}{l}\text { Campos et } \\
\text { al., } 2008\end{array}$ & 28 & $\begin{array}{l}\text { Indivíduos usuários de IC } \\
\text { nas habilidades de } \\
\text { ordenação temporal. }\end{array}$ & $\begin{array}{l}\text { Utilização dos testes TPD e } \\
\text { TPF. }\end{array}$ & $\begin{array}{l}\text { Usuários de IC apresentaram } \\
\text { semelhante desempenho nos } \\
\text { dois testes quando comparados } \\
\text { ao grupo de indivíduos com } \\
\text { audição normal. }\end{array}$ \\
\hline $\begin{array}{l}\text { Samelli, } \\
\text { Schochat, } \\
2008\end{array}$ & 100 & $\begin{array}{l}\text { Respostas das orelhas } \\
\text { direita e esquerda no } \\
\text { teste GIN. }\end{array}$ & Utilização do Teste GIN. & $\begin{array}{l}\text { Não houve vantagem de uma } \\
\text { orelha sobre a outra no teste } \\
\text { GIN. }\end{array}$ \\
\hline $\begin{array}{l}\text { Sanches et } \\
\text { al., } 2008\end{array}$ & 40 & $\begin{array}{l}\text { Processamento auditivo } \\
\text { em idosos que relatam } \\
\text { audição normal. }\end{array}$ & $\begin{array}{l}\text { Exame otorrinolaringológico, } \\
\text { avaliação audiológica } \\
\text { básica, testes: PSI/SSI, TPF } \\
\text { e SSW. }\end{array}$ & $\begin{array}{l}\text { Os resultados apontaram para } \\
\text { ineficiência das funções } \\
\text { auditivas centrais em indivídous } \\
\text { idosos que relatam ouvir bem. }\end{array}$ \\
\hline $\begin{array}{l}\text { Boscariol et } \\
\text { al., } 2009\end{array}$ & 20 & $\begin{array}{l}\text { Crianças com fissura } \\
\text { isolada de palato em } \\
\text { testes do processamento } \\
\text { auditivo. }\end{array}$ & $\begin{array}{l}\text { Utilização dos testes AFT-R, } \\
\text { PSI/SSI, SSW e DD. }\end{array}$ & $\begin{array}{l}\text { Os piores desempenhos } \\
\text { apresentados pelas crianças } \\
\text { foram nos testes AFT-R, DD, } \\
\text { SSW e PSI/SSI. }\end{array}$ \\
\hline $\begin{array}{l}\text { Balen et al., } \\
2009\end{array}$ & 31 & $\begin{array}{l}\text { Resolução temporal entre } \\
\text { crianças com audição } \\
\text { normal, perda auditiva } \\
\text { condutiva e distúrbios do } \\
\text { processamento auditivo. }\end{array}$ & $\begin{array}{l}\text { Utilização de questionário } \\
\text { aos responsáveis, avaliação } \\
\text { audiológica e do } \\
\text { processamento auditivo } \\
\text { (DD, FF, SSW e RGDT). }\end{array}$ & $\begin{array}{l}\text { A perda auditiva condutiva e o } \\
\text { distúrbio do processamento } \\
\text { auditivo exercem influência no } \\
\text { desempenho do teste RGDT. }\end{array}$ \\
\hline
\end{tabular}

Legenda: TPD= Teste Padrão de Duração; TPF= Teste Padrão de Frequência; SSW= Staggered Spondaic Word Test; DD= Dicótico de Dígitos; AFT-R= Auditory Fusion Test-Revised; LS= Localização Sonora; DNV= Dicótico não-verbal; IC= Implante coclear; GIN= Gaps in Noise; PSI/SSI= Teste de identificação de sentenças sintéticas e FF= Fala Filtrada.

\section{Figura2-Publicações sobreaspectos temporais auditivos na Revista Brasileira de Otorrinolaringologia}

A Figura 4 resume o tema, os métodos utilizados e as conclusões desses estudos.

O levantamento realizado nesta pesquisa permitiu verificar que existe um número reduzido de estudos nacionais relacionados ao tema sobre aspectos temporais auditivos. Considerando-se somente os quatro periódicos nacionais, menos de $9 \%(8,54 \%)$ das produções científicas abordaram essa temática.

Dentro dos estudos levantados nessa pesquisa, foi observada a preferência do fonoaudiólogo por estudar os aspectos temporais auditivos em crianças 1-3,6-8,14,15,18,19,21,22,25,26,29,32, seguida por estudos com adultos 5,9,11,13,20,23,30,31 e finalmente por idosos ${ }^{23,27}$.

Em relação a resolução temporal, os testes mais usados nas pesquisas nacionais foram o GIN ${ }^{3,12-14,16,21}$ e o RGDT 1,3,5,6,9,13,21,22,23 e correlacionaram os aspectos temporais ao desenvolvimento normal ${ }^{1,3,6,9,12-14,21-23}$, dificuldades escolares ${ }^{15} \mathrm{e}$ alterações de linguagem ${ }^{1,6,22}$.

Quanto a ordenação temporal, o presente estudo revelou que a maioria utilizam os testes TPF $5,10,11,17-20,27,29$ e TPD 7,11,16,17,19,20,30. Há estudos 


\begin{tabular}{|c|c|c|c|c|}
\hline & $\mathbf{N}$ & Tema & Métodos & Conclusões \\
\hline $\begin{array}{l}\text { Da Silva et } \\
\text { al., } 2006\end{array}$ & 20 & $\begin{array}{l}\text { Processamento auditivo } \\
\text { em operadores de } \\
\text { telemarketing }\end{array}$ & $\begin{array}{l}\text { Utilização de questionário e } \\
\text { dos testes: FF, RGDT e } \\
\text { MLD.. }\end{array}$ & $\begin{array}{l}\text { As habilidades de interação } \\
\text { binaural e resolução temporal } \\
\text { apresentaram-se alteradas em } \\
\text { parte dos indivíduos. }\end{array}$ \\
\hline $\begin{array}{l}\text { Muniz et al., } \\
2007\end{array}$ & 36 & $\begin{array}{l}\text { Resolução temporal (tom } \\
\text { puro) em crianças com } \\
\text { desvio fonológico. }\end{array}$ & Realização do RGDT. & $\begin{array}{l}\text { As crianças com desvio } \\
\text { fonológico apresentaram um } \\
\text { limiar maior quando comparadas } \\
\text { às crianças sem desvio } \\
\text { fonológico. Não houve influência } \\
\text { das variáveis: frequência, } \\
\text { gênero, idade, série escolar ou } \\
\text { ocorrência de tratamento } \\
\text { fonoaudiológico. }\end{array}$ \\
\hline $\begin{array}{l}\text { Samelli, } \\
\text { Schochat, } \\
2008\end{array}$ & & $\begin{array}{l}\text { Revisão teórica sobre } \\
\text { processamento auditivo e } \\
\text { resolução temporal. }\end{array}$ & $\begin{array}{l}\text { Pesquisa bibliográfica em } \\
\text { periódicos nacionais e } \\
\text { internacionais, livros e } \\
\text { documentos eletrônicos. }\end{array}$ & $\begin{array}{l}\text { O processamento auditivo e a } \\
\text { resolução temporal são } \\
\text { fundamentais para aquisição da } \\
\text { linguagem. }\end{array}$ \\
\hline $\begin{array}{l}\text { Balen et al., } \\
2008\end{array}$ & 73 & $\begin{array}{l}\text { Resolução temporal de } \\
\text { crianças escolares. }\end{array}$ & $\begin{array}{l}\text { Realização dos testes GIN e } \\
\text { RGDT. }\end{array}$ & $\begin{array}{l}\text { Possibilidade dos dois testes } \\
\text { não avaliarem a mesma } \\
\text { habilidade ou requisitarem } \\
\text { processos não auditivos nas } \\
\text { tarefas solicitadas, uma vez que } \\
\text { há diferenças dos limiares dos } \\
\text { testes. }\end{array}$ \\
\hline $\begin{array}{l}\text { Schochat et } \\
\text { al., } 2009\end{array}$ & 155 & $\begin{array}{l}\text { Potenciais evocados } \\
\text { auditivos de média } \\
\text { latência e ordenação } \\
\text { temporal. }\end{array}$ & $\begin{array}{l}\text { Realização dos testes TPD, } \\
\text { TPF e PEAML. }\end{array}$ & $\begin{array}{l}\text { Não houve concordância entre } \\
\text { os três testes. }\end{array}$ \\
\hline $\begin{array}{l}\text { Santos et al., } \\
2010\end{array}$ & 12 & $\begin{array}{l}\text { Ordenação e resolução } \\
\text { temporal em crianças } \\
\text { com desvio fonológico. }\end{array}$ & $\begin{array}{l}\text { Utilização dos testes PCC, } \\
\text { RGDT e PPS. }\end{array}$ & $\begin{array}{l}\text { As crianças com desvio } \\
\text { fonológico apresentaram } \\
\text { desempenho adequado nos } \\
\text { testes de processamento } \\
\text { temporal, contudo existe uma } \\
\text { relação estatisticamente } \\
\text { significante entre a severidade } \\
\text { do desvio e o desempenho no } \\
\text { teste PPS. }\end{array}$ \\
\hline
\end{tabular}

Legenda: FF= Fala Filtrada; RGDT= Random Gap Detection Test; MLD= Mask Level Difference; Gin= Gaps in Noise; TPD= Teste Padrão de Duração; TFP ou PPS= Teste Padrão de Frequência; PEAML= Potenciais Evocados Auditivos de Média Latência e PCC= Porcentagem de Consoantes Corretas.

Figura 3 - Publicações sobre aspectos temporais auditivos na Revista CEFAC

buscando correlações com: desempenho escolar ${ }^{10}$, desenvolvimento de linguagem ${ }^{18,29}$. Cabe ressaltar também a presença de estudos que discutem a aplicação e efetividade do uso dos testes na prática clínica ${ }^{19,22}$.

Nota-se a ausência de produções que abordam a terapia para pacientes com déficits nas habilidades dos aspectos temporais, contratando com a pluralidade de estudos que avaliam a resolução e/ou ordenação temporais em indivíduos ouvintes. 1,3,5-8, 11-15,18,19,21-23,25,26,29,30,32.

Além disso, pode ser observado que, ainda que existam estudos realizados por diferentes autores sobre determinado tema, não há normatização quanto aos resultados esperados nos diversos testes que avaliam a Resolução Temporal Auditiva, nem mesmo um consenso sobre qual teste deveria ser utilizado, uma vez que existem inúmeras variáveis ${ }^{3,25}$.

Há poucos centros no país dedicados a estudos sobre os aspectos temporais auditivos, sendo que esses se encontram em fase inicial em suas pesquisas ${ }^{1}$.

Vale ressaltar que, foi percebida a presença de alguns trabalhos que possuíam escassa análise estatística. Porém, foram vislumbrados diversos estudos melhores detalhados, proporcionando aos leitores dados que realmente são analisáveis. 


\begin{tabular}{|c|c|c|c|c|}
\hline & $\mathbf{N}$ & Tema & Métodos & Conclusões \\
\hline Elias, 2009 & & $\begin{array}{l}\text { Habilidades do } \\
\text { processamento auditivo } \\
\text { central em crianças e } \\
\text { adolescentes com } \\
\text { doença cerebrovascular. }\end{array}$ & $\begin{array}{l}\text { Aplicação da Avaliação } \\
\text { Simplificada do } \\
\text { Processamento Auditivo e } \\
\text { dos testes: FR, FF, DNV, } \\
\text { DCV, DD, SSW, TPF e TPD. }\end{array}$ & $\begin{array}{l}\text { Os indivíduos com DCV } \\
\text { apresentaram desempenho } \\
\text { inferior em todos os testes se } \\
\text { comparados ao grupo controle, } \\
\text { com exceção dos testes de } \\
\text { Localização Sonora e FR. }\end{array}$ \\
\hline $\begin{array}{l}\text { Queiroz et } \\
\text { al., } 2009\end{array}$ & 72 & $\begin{array}{l}\text { Desempenho de } \\
\text { mulheres e jovens idosas } \\
\text { no RGDT. }\end{array}$ & Utilização do RGDT. & $\begin{array}{l}\text { O avanço da idade interfere } \\
\text { negativamente no desempenho } \\
\text { no RGDT. }\end{array}$ \\
\hline $\begin{array}{l}\text { Elgemmann, } \\
\text { Ferreira, } \\
2009\end{array}$ & 30 & $\begin{array}{l}\text { Processamento auditivo } \\
\text { em crianças com } \\
\text { dificuldades de } \\
\text { aprendizagem. }\end{array}$ & $\begin{array}{l}\text { Aplicação de testes de } \\
\text { leitura e escrita, ASPA, DD, } \\
\text { SSW e PPS. }\end{array}$ & $\begin{array}{l}\text { Todos os participantes } \\
\text { apresentaram transtorno do } \\
\text { processamento auditivo em } \\
\text { quase todos os subperfis } \\
\text { primários. }\end{array}$ \\
\hline $\begin{array}{l}\text { Balen et al., } \\
2010\end{array}$ & 44 & $\begin{array}{l}\text { Influência do nível } \\
\text { socioeconômico na } \\
\text { resolução temporal em } \\
\text { escolares. }\end{array}$ & $\begin{array}{l}\text { Realização dos testes GIN e } \\
\text { RGDT. }\end{array}$ & $\begin{array}{l}\text { Houve influência do nível } \\
\text { socioeconômico no desempenho } \\
\text { dos dois testes. }\end{array}$ \\
\hline
\end{tabular}

Legenda: FR= Fala com Ruído; FF= Fala Filtrada; DNV= Dicótico Não-verbal; DCV= Dicótico Consoante-vogal; DD- Dicótico de Dígitos, SSW= Staggered Spondaic Word Test; TPF ou PPS= Teste Padrão de Frequência; TPD= Teste Padrão de Duração; RGDT= Random Gap Detection Test; ASAPA= Avaliação Simplificada do Processamento Auditivo e GIN= Gaps in Noise.

\section{Figura 4 - Publicações sobre aspectos temporais auditivos na Revista Sociedade Brasileira de} Fonoaudiologia

Este trabalho utilizou somente artigos publicados nacionalmente, visando ampliar a divulgação das pesquisas realizadas no Brasil a respeito de um tema tão importante, possibilitando um contato mais próximo entre a produção e a aplicação do conhecimento.

\section{CONCLUSÃO}

Na última década, o número de estudos de revisão bibliográfica na área de Fonoaudiologia está aumentando. Embora ainda seja pouco abundante, esse tipo de produção científica possui importância certificada na área da saúde em geral.

As produções científicas encontradas na presente pesquisa, em sua maioria, reforçam a participação do fonoaudiólogo junto à equipe profissional atuante nas escolas no que se refere à orientação e ao encaminhamento, como também no fornecimento de conhecimentos próprios ao ambiente escolar, como a possibilidade de terapia para crianças com alteração nos aspectos temporais auditivos.

\section{ABSTRACT}

Background: this article was produced based on a review of articles published since 2005 in four major national journals in the fields of Speech (Pró-Fono Revista de Atualização Científica, Revista Brasileira de Otorrinolaringologia, Revista CEFAC and Revista Sociedade Brasileira de Fonoaudiologia) on the temporal aspects of hearing. This study points out to three addressed issues: temporal aspects, temporal ordering and temporal resolution. Purpose: to delineate the profile of knowledge production in the area of auditory temporal aspects with reference to four national journals. Conclusion: the survey conducted in this research showed that there is a small number of national studies related to the issue concerning the temporal aspects of hearing. Considering only the four national journals, less than $9 \%$ of the scientific production addressed this issue.

KEYWORDS: Hearing; Auditory Perception; Hearing Tests 


\section{REFERÊNCIAS}

1. Muniz LF, Roazzi A, Schochat E, Teixeira CF, de Lucena, JA. Avaliação da habilidade de resolução temporal, com uso do tom puro, em crianças com e sem desvio fonológico. Rev CEFAC. 2007; 9(4): 550-62.

2. Cassab, TV, Zorzetto NL. Teste da fusão auditiva-revisado (AFT-R) em crianças com fissura labiopalatina. ACTA ORL/Técnicas em Otorrinolaringologia. 2006; 24(4): 272-6.

3. Balen SA, Liebel G, Boeno MR, Mottecy CM. Resolução temporal de crianças escolares. Rev CEFAC. 2008; 11(supl. 1): 52-61.

4. Albuquerque DBL. Compreensão de leitura e habilidades de processamento auditivo em crianças [dissertação mestrado] Recife (PE): Universidade Católica de Pernambuco; 2007.

5. Ishii C, Arashiro PM, Pereira LD. Ordenação e resolução temporal em cantores profissionais e amadores afinados e desafinados. Pró-Fono Revista de Atualização Científica. 2006; 18 (3): 285-92.

6. Balen SA, Bretzke LM, Mottecy CM, Liebel G, Boeno MR, Gondim LM. Resolução temporal de crianças: comparação entre audição normal, perda auditiva condutiva e distúrbio do processamento auditivo. Rev Bras Otorrinolaringol. 2009; 75(1): 123-9.

7. Ramos CS, Pereira LD. Processamento auditivo e audiometria de altas freqüências em escolares de São Paulo. Pró-Fono Revista de Atualização Científica. 2005; 17 (2): 153-64.

8. Boscariol M, André KD, Feniman MR. Crianças com fissura isolada de palato: desempenho nos testes de processamento auditivo. Rev Bras Otorrinolaringol. 2009; 75 (2): 213-20.

9. da Silva MC, Cunha MB, Souza CC, Mitre El. Avaliação do processamento auditivo em operadores de telemarketing. Rev CEFAC. 2006; 8(4): 536-42.

10. Elgelmann L, Ferreira MDC. Avaliação do processamento auditivo em crianças com dificuldades de aprendizagem. Rev Soc Bras Fonoaudiol. 2009; 14 (1): 69-74.

11. Onoda RM, Pereira LD, Guilherme A. Reconhecimento de padrão temporal e escuta dicótica em descendentes de japoneses, falantes e não-falantes da língua japonesa. Rev Bras Otorrinolaringol. 2006; 72 (6): 737-46.

12. Samelli AG, Schochat E. Estudo da vantagem da orelha em teste de detecção de gap. Rev Bras Otorrinolaringol. 2008; 74(2): 235-40.

13. Zaidan E, Garcia AP, Tedesco MLF, Baran JA. Desempenho de adultos jovens normais em dois testes de resolução temporal. Pró-Fono Revista de Atualização Científica. 2008; 20(1): 19-24.

14. Perez AP, Pereira LD. O Teste Gap in Noise em crianças de 11 e 12 anos. Pró-Fono Revista de Atualização Científica. 2010; 22 (1): 7-12.

15. Santos JLF, Parreira LMMV, Leite RCD. Habilidades de ordenação e resolução temporal em crianças com desvio fonológico. Rev CEFAC. 2010;12(3):371-6.

16. Liporaci FD. Estudo do processamento auditivo temporal (resolução e ordenação) em idosos [dissertação mestrado] Rio de Janeiro (RJ): Universidade Veiga de Almeida; 2009.

17. Frota S, Pereira LD. Processos temporais em crianças com déficit de consciência fonológica. Revista Iberoamericana de Educación. 2006; 1 - 11. 18. Fortunato-Tavares $T$, Rocha $C$, Furquim $C A$, Befi-Lopes D, Schochat E, Hestvik A, Schwartz R. Processamento Lingüístico e Processamento Auditivo Temporal em Crianças com Distúrbio Específico de Linguagem. Pró-Fono Revista de Atualização Científica. 2009; 21 (4): 279-84.

19. Schochat $E$ et al. Processamento auditivo: comparação entre potenciais evocados auditivos de média latência e testes de padrões temporais. Rev CEFAC. 2009; 11 (2): 314-22.

20. Campos PD et al. Habilidades de ordenação temporal em usuários de implante coclear multicanal. Rev Bras Otorrinolaringol. 2008; 74 (6): 884-9.

21. Balen AS, Boeno MRM, Liebel, G. A influência do nível socioeconômico na resolução temporal em escolares. Rev Soc Bras Fonoaudiol. 2010; 15(1): 7-13.

22. Fortes AB, Pereira LD, Azevedo MF. Resolução temporal: análise em pré-escolares nascidos a termo e pré-termo. Pró-Fono Revista de Atualização Científica. 2007; 19(1): 87-96.

23. Queiroz DS, Branco-Barreiro FCA, MomensohnSantos TM. Desempenho no Teste de Detecção de Intervalo Aleatório - Random Gap Detection Test (RGDT): estudo comparativo entre mulheres jovens e idosas. Rev Soc Bras Fonoaudiol. 2009; 14 (4): 503-7.

24. Samelli AG. O Teste GIN (Gap in noise): limiares de detecção de gap em adultos com audição normal [dissertação doutorado] São Paulo (SP): Faculdade de Medicina da Universidade de São Paulo; 2005.

25. Murphy CF, Schochat E. Influência de paradigmas temporais em testes de processamento temporal auditivo. Pró-Fono Revista de Atualização Científica. 2007; 19(3): 259-66.

26. Elias KMIF, Santos MFC, Ciasca SM, MouraRibeiro MVL. Processamento auditivo em criança com doença cerebrovascular. Pró-Fono Revista de Atualização Científica. 2007; 19 (4): 393-400. 
27. Sanchez LM, Nunes FB, Barros F, Ganança $\mathrm{MM}$, Capovilla HH. Avaliação do processamento auditivo em idosos que relatam ouvir bem. Rev Bras Otorrinolaringol . 2008; 74(6): 896-902.

28. Elias KMIF. Doença cerebrovascular na infância e adolescência: estudo das habilidades de processamento auditivo (central). Rev Soc Bras Fonoaudiol. 2009; 14 (1): 152.

29. Abdo A, Murphy C, Schochat E. Habilidades auditivas em crianças com dislexia e transtorno do déficit de atenção e hiperatividade. Pró-Fono Revista de Atualização Científica. 2010; 22 (1): 25-30.
30. Meneguello J, Leonhardt DF, Pereira LD. Processamento auditivo em indivíduos com epilepsia de lobo temporal. Rev Bras Otorrinolaringol. 2006; 72(4): 496-504.

31. Samelli AG, Schochat E. Processamento auditivo, resolução temporal e teste de detecção de gap: revisão de literatura. Rev CEFAC. 2008; 10(3): 369-77.

32. Murphy CFB, Schochat E. Correlações entre leitura, consciência fonológica e processamento temporal auditivo. Pró-Fono Revista de Atualização Cientifica. 2009; 21(1): 13-8.

Endereço para correspondência:

Sulamita da Silva Marcelino Terto

Rua Itaguara, 196 - apto 302 - Floresta

Belo Horizonte - Minas Gerais, MG

CEP: $31110-240$

E-mail: sulamitamarcelino@yahoo.com.br 